\title{
Efficacy and safety of a novel synergistic drug candidate, CRx-102, in hand osteoarthritis
}

\author{
T K Kvien, ${ }^{1,2}$ E Fjeld, ${ }^{3}$ B Slatkowsky-Christensen, ${ }^{1}$ M Nichols, ${ }^{4}$ Y Zhang, ${ }^{4}$ A Prøven, ${ }^{3}$ \\ K Mikkelsen, ${ }^{5} \varnothing$ Palm, ${ }^{6}$ A A Borisy, ${ }^{4}$ J Lessem $^{4}$
}

${ }^{1}$ Department of Rheumatology, Diakonhjemmet Hospital, Oslo, Norway: ${ }^{2}$ Faculty of Medicine, University of Oslo, Norway;

${ }^{3}$ Department of Rheumatology, Martina Hansens Hospital, Sandvika, Norway;

${ }^{4}$ CombinatoRx Inc., Cambridge, Massachusetts, USA;

${ }^{5}$ Department of Rheumatology, Lillehammer Hospital for Rheumatic Diseases,

Lillehammer, Norway;

${ }^{6}$ Department of Rheumatology, Østfold Hospital, Sarpsborg,

Norway

Correspondence to:

Professor T K Kvien, Department of Rheumatology,

Diakonhjemmet Hospital, Box 23

Vinderen, N-0319 Oslo, Norway;

t.k.kvien@medisin.uio.no

Accepted 2 October 2007 Published Online First 25 October 2007

\section{ABSTRACT}

Objective: The novel synergistic drug candidate CRx-102 comprises dipyridamole and low dose prednisolone and is in clinical development for the treatment of immunoinflammatory diseases. The purpose of this clinical study was to examine the efficacy and safety of CRx-102 in patients with hand osteoarthritis (HOA).

Methods: The study was conducted as a blinded, randomised, placebo-controlled trial at four centres in Norway. Eligibility criteria included being of age 30-70 years, at least one swollen and tender joint, a KellgrenLawrence $(\mathrm{K}-\mathrm{L})$ score of 2 or higher on radiographs, and a score of at least $30 \mathrm{~mm}$ pain on the Australian/Canadian Osteoarthritis Hand Index (AUSCAN) visual analogue pain scale (VAS). The primary endpoint was a reduction in pain from baseline to day 42 on the AUSCAN pain subscale. Two-sided $p$ values for the differences in least squares (LS) means adjusted for baseline are presented.

Results: The mean age of the 83 patients with HOA was 60 years and $93 \%$ were females. CRx-102 was statistically superior to placebo at 42 days for changes in AUSCAN pain (LS mean -14.2 vs -4.0 ) and for clinically relevant secondary endpoints (joint pain VAS (-18.6 vs -6.3), patient global VAS $(-15.9$ vs -4.2$)$ ) in the intention to treat population. The most frequently reported adverse event during the study was headache $152 \%$ in CRx-102 vs $15 \%$ in the placebo group).

Conclusions: The novel synergistic drug candidate CRx102 demonstrated efficacy by statistically reducing pain compared to placebo in HOA and was generally well tolerated.

Osteoarthritis (OA) is the most common rheumatic joint disease. It is reported to be more prevalent than all other forms of arthritis, and this prevalence seems to be increasing. ${ }^{1}$ The typical clinical manifestations are pain, stiffness and physical disability. Localised loss of hyaline articular cartilage and adjacent bone remodelling are the key structural changes of $\mathrm{OA}$, and local inflammation may also contribute to the pain and joint damage. $^{2}$

Knee $\mathrm{OA}$ is the most common form of the disease, followed by hand $\mathrm{OA}(\mathrm{HOA}){ }^{3}$ The majority of people aged 55 years and over have radiographic changes of $\mathrm{OA}$ in at least one hand joint and approximately one-fifth of this population has symptomatic HOA. ${ }^{45}$ The prevalence of $\mathrm{HOA}$ increases with age and is higher in females than in males. ${ }^{4}$ Recent studies also indicate that the burden of disease for patients with HOA is considerable across a variety of dimensions of health-related quality of life. ${ }^{6}$
Non-steroidal anti-inflammatory drugs (NSAIDs), including selective cyclo-oxygenase-2 (COX-2) inhibitors are important symptommodifying therapeutic options for patients with OA. ${ }^{7-10}$ However, NSAIDs are associated with risk of gastrointestinal adverse events and the selective COX-2 inhibitors have come under special scrutiny because of cardiovascular adverse effects. ${ }^{11}{ }^{12}$ Similar concerns have recently been raised about the cardiovascular safety of non-naproxen NSAIDs, ${ }^{13}$ and the efficacy of long-term dosing of NSAIDs in knee OA has also been questioned. ${ }^{14}$ Few controlled clinical trials have addressed the efficacy of pharmacological therapies in HOA and, in particular, few controlled studies have included a placebo group. ${ }^{10}{ }^{15}$ Thus, there is an obvious need to document the efficacy of existing drugs and, in particular, to identify new and effective agents for patients with $\mathrm{HOA} .^{16}$

Corticosteroids are a mainstay of effective antiinflammatory therapy in many clinical settings, but the side effects associated with chronic administration have limited their use in OA to occasional intra-articular administration. It has long been a goal to develop a therapeutic agent with the anti-inflammatory and disease modifying activities of corticosteroids without their associated side effects. ${ }^{17}$ One approach to creating such a therapeutic agent is to develop a drug combination that contains a glucocorticoid and an enhancing agent that pair synergistically to generate a powerful anti-inflammatory effect. Synergistic combinations can have an effect that is greater and more selective than the sum of the activities of the individual components, and thus can provide greater therapeutic benefit with lower toxicity. Synergy is often observed in multi-target therapeutics that modulate the activity of two or more molecular targets to create a novel therapeutic action. ${ }^{18}{ }^{19}$ In vivo models testing anti-inflammatory combinations containing a low dose steroid have demonstrated a synergistic interaction between the steroid and the enhancing agent that produces an effect equivalent to that of a high dose steroid alone, without indications of high dose steroid side effects.

CRx-102 is one such novel synergistic drug candidate and is in clinical development for the treatment of immunoinflammatory diseases including rheumatoid arthritis (RA) and OA. This drug candidate comprises a combination of a low dose of prednisolone $(3 \mathrm{mg}$ ) and 200 or $400 \mathrm{mg}$ dipyridamole. According to results from pre-clinical pharmacology experiments, CRx-102 works through a novel mechanism of action by which online under the BMJ Journals unlocked scheme, see http:// ard.bmi.com/info/unlocked.dtl 
dipyridamole selectively amplifies prednisolone's anti-inflammatory and immunomodulatory effects without replicating steroid side effects. ${ }^{20-22}$ The objective of the current phase 2 study was to evaluate the efficacy and safety of the novel synergistic drug candidate CRx-102 compared to placebo in patients with HOA over a 6-week dosing period.

\section{MATERIALS AND METHODS}

\section{Study population}

Males and females between the ages of 30 and 70 years with $\mathrm{HOA}$, as defined by the American College of Rheumatology (ACR) criteria, ${ }^{23}$ were enrolled in the study. Additional inclusion criteria included presence of more than one swollen joint and more than one tender joint, a Kellgren-Lawrence (K-L) score of two or more on radiographs and self-reported hand pain that had to be at least $30 \mathrm{~mm}$ on the Australian/Canadian Osteoarthritis Hand Index (AUSCAN) visual analogue scales (VAS). ${ }^{24}{ }^{25}$ All subjects had to sign and date an informed consent. The regional ethics committee evaluated the study, the storage and analyses of data was licensed from the data inspectorate and approval for the collection of biologic material was obtained from the Department of Health.

Subjects who were pregnant, lactating or using hormonal birth control pills as well as subjects with a history of hypersensitivity to corticosteroids and/or dipyridamole, taking bisphosphonates or who had a positive rheumatoid factor test were excluded from the study. Furthermore, subjects who had taken any corticosteroids orally, topically or intra-articularly 3 months prior to enrolment were also excluded. Other exclusion criteria included a history of asthma, HIV infection, hepatitis, currently uncontrolled diabetes, use of statins in a dose that had changed during the prior 3 months, known active infection or a surgical procedure within 30 days of study initiation. Since one of the components of CRx-102 is dipyridamole, patients on warfarin, ticlopidine, clopidogrel or aspirin of more than $81 \mathrm{mg}$ daily were also excluded from entering the study.

\section{Design and medication}

The study was designed as a 6-week randomised, blinded, placebo-controlled four-centre parallel group study. Within 2 weeks of a screening visit, patients who fulfilled the eligibility criteria were randomly assigned to CRx-102 or placebo. Followup assessments were performed after 7, 14, 28 and 42 days, with a final safety visit after approximately 56 days.

A total of 83 subjects were randomised 1:1 to a daily dose regimen of either CRx-102 or placebo. CRx-102 for days 1-7 combined $2 \mathrm{mg}$ of prednisolone with $100 \mathrm{mg}$ of dipyridamole at $8 \mathrm{am}$ and $1 \mathrm{mg}$ of prednisolone with $100 \mathrm{mg}$ of dipyridamole at $1 \mathrm{pm}$. From days 8-42 CRx-102 combined $2 \mathrm{mg}$ prednisolone with $200 \mathrm{mg}$ of dipyridamole at $8 \mathrm{am}$ and $1 \mathrm{mg}$ of prednisolone
Figure 1 Flow chart of the selection of patients for this study and patient disposition.

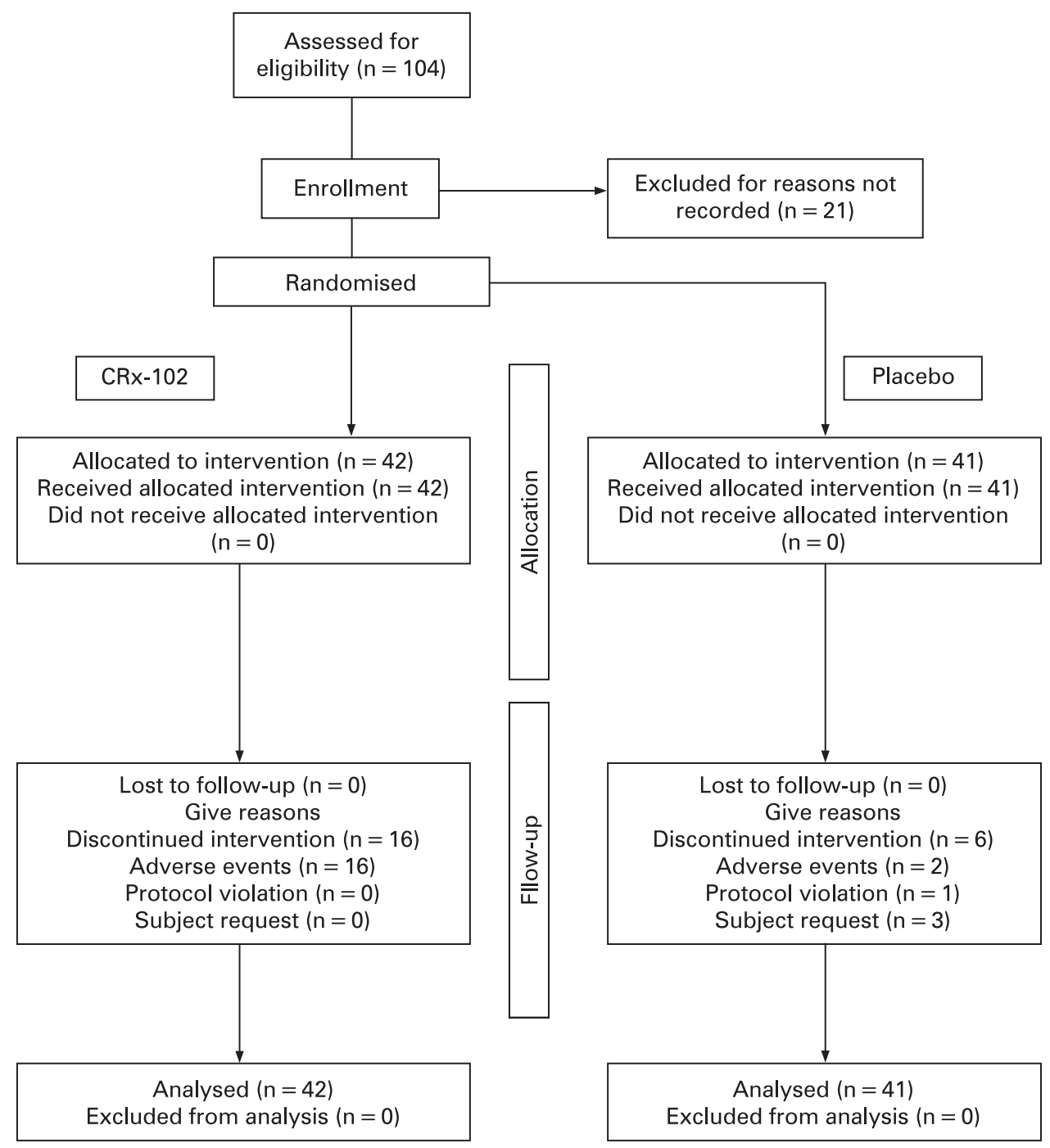


with $200 \mathrm{mg}$ dipyridamole at $1 \mathrm{pm}$. Patients in the placebo group received an equal number of tablets, dosed at the same time of day as for the test compound and all containing placebo.

Paracetamol was provided as rescue medication throughout the study at a daily dose of up to $4000 \mathrm{mg}$, and the usage was recorded. The use of NSAIDs in all subjects was stopped for the duration of the study starting at the initial screening visit.

\section{Assessments}

The AUSCAN ${ }^{24} 25$ was used as the primary assessment tool. Previous studies have demonstrated that this instrument has acceptable reliability, construct validity and responsiveness. The translated Norwegian version has also satisfactory clinimetric properties. ${ }^{26}$ We chose to use the version with responses on VAS to each item. AUSCAN has five items measuring pain, one measuring stiffness and nine measuring physical function. The pain and physical functioning scores were normalised to a 0-100-point scale prior to the analyses.

Additional patient-reported measures included a joint pain VAS (question: how would you describe the intensity of your joint pain during the last 2 days?) and global assessment VAS (question: we ask you to evaluate the activity of your osteoarthritis over the last 2 days. When you take all symptoms into consideration, how will you evaluate your condition?). The patients did not have access to scores from previous visits when they were performing each subsequent assessment.

The patients were clinically examined for vital signs at each visit and each individual finger joint (distal interphalangeal (DIP), proximal interphalangeal (PIP), metacarpophalangeal (MCP) and carpometacarpal (CMC)) on the right and left hand was examined for the presence of joint tenderness, soft tissue swelling, bony enlargement and limited joint motion. A score was calculated using the number of PIP and DIP joints for the presence of each of these four characteristics (ie, score range 0 18 for each).

Sera were frozen and stored and later analysed with a high sensitivity technique to determine levels of C-reactive protein (CRP). Regular blood chemistry, including fasting blood glucose, was recorded. Unsolicited adverse events were also recorded.

\section{Analyses}

AUSCAN pain was the predefined primary endpoint. The sample size was calculated based on an assumed improvement of $20 \%$ in AUSCAN pain VAS in the CRx-102 group compared to a $10 \%$ improvement in the placebo-group from baseline to
Table 1 Demographic variables and joint involvement (mean (SD)) for continuous variables, percentages for counts)

\begin{tabular}{lll}
\hline & CRx-102 (n = 42) & Placebo (n = 41) \\
\hline Age & $61.1(5.0)$ & $59.6(5.3)$ \\
Female & 93 & 93 \\
Caucasian & 100 & 100 \\
Height, cm & $166.0(6.7)$ & $167.7(8.2)$ \\
Weight, kg & $71.1(12.0)$ & $74.5(14.6)$ \\
Percentage with OA joint & & \\
involvement: & & \\
Right MTP joint I & 36 & 10 \\
Left MTP joint I & 33 & 10 \\
Lumbar spine & 24 & 17 \\
Cervical spine & 19 & 7 \\
Right hip & 17 & 10 \\
Left hip & 10 & 12 \\
Right knee & 19 & 7 \\
Left knee & 17 & 10 \\
Other joints & 17 & 15 \\
Finger joints: percentage with & & \\
radiographic grade 2-4 K-L score: & & \\
Minimal (2) & 14 & 12 \\
Moderate (3) & 45 & 32 \\
Severe (4) & 40 & 56 \\
\hline
\end{tabular}

K-L, Kellgren-Lawrence; MTP, metatarsophalangeal; OA, osteoarthritis.

day 42 with an alpha of $0.05 \%$, to achieve $80 \%$ power assuming a $15 \%$ drop out rate using a one tailed $t$ test for the comparison of the mean changes.

The primary analysis was conducted on the intention to treat (ITT) population that included all patients who took at least one dose of study medication. Secondarily, an analysis was performed in the per-protocol population, which was defined as all subjects who received at least one dose of study medication, had no major protocol violations and had a study drug compliance of at least $75 \%$. The treatment effects were derived from analysis of covariance (ANCOVA) adjusting for the baseline values. These analyses tested the difference (with 95\% confidence intervals) between these adjusted mean changes in the active drug compared to the placebo group from baseline to 42 days. The last non-missing post-baseline observation was carried forward (LOCF) to replace subsequent missing values. Two-sided $p$ values for the differences in least square means adjusted for baseline are presented (the study protocol recommended use of one-sided tests, but we found it more

Table 2 Baseline mean (SD) values of efficacy variables, adjusted mean changes from baseline to day 42 (least squares mean (standard error of mean)) and treatment effect (mean difference (95\% CI) placebo minus CRx-102) in the intention to treat population

\begin{tabular}{|c|c|c|c|c|c|c|}
\hline & \multicolumn{2}{|l|}{ Baseline } & \multicolumn{2}{|l|}{ Changes } & \multirow[b]{2}{*}{ Treatment effect } & \multirow[b]{2}{*}{ p Value } \\
\hline & CRx-102 (n = 42) & Placebo $(n=41)$ & CRx-102 & Placebo & & \\
\hline \multicolumn{7}{|l|}{ AUSCAN: } \\
\hline Pain & $57.9(20.2)$ & $60.9(19.4)$ & $-14.2(3.0)$ & $-4.0(3.1)$ & $10.2(1.6$ to 18.7$)$ & 0.020 \\
\hline Physical & $62.4(19.5)$ & $67.8(17.5)$ & $-8.1(2.7)$ & $-3.6(2.7)$ & $4.5(-3.2$ to 12.2$)$ & 0.246 \\
\hline Stiffness & $61.1(18.0)$ & $64.5(21.2)$ & $-15.2(3.2)$ & $-7.7(3.3)$ & $7.5(-1.7$ to 16.7$)$ & 0.108 \\
\hline \multicolumn{7}{|l|}{ VAS: } \\
\hline Joint pain & $58.3(20.1)$ & $62.1(16.9)$ & $-18.6(3.3)$ & $-6.3(3.3)$ & $12.3(3.0$ to 21.5$)$ & 0.010 \\
\hline \multicolumn{7}{|l|}{ Lab tests: } \\
\hline CRP mg/litre & $2.5(2.9)$ & $2.3(2.2)$ & $-0.2(0.4)$ & $0.1(0.4)$ & $0.3(-0.7$ to 1.4$)$ & 0.536 \\
\hline \multicolumn{7}{|l|}{ Joint counts: } \\
\hline Tender joints & $9.5(4.7)$ & $9.4(4.6)$ & $-3.6(0.7)$ & $-2.4(0.7)$ & $1.2(-0.9$ to 3.2$)$ & 0.258 \\
\hline Soft tissue swelling & $5.5(4.7)$ & $5.0(4.4)$ & $-2.4(0.5)$ & $-1.6(0.5)$ & $0.8(-0.6$ to 2.2$)$ & 0.262 \\
\hline
\end{tabular}

AUSCAN, Australian/Canadian Osteoarthritis Hand Index; CRP, C-reactive protein; VAS, visual analogue scale. 

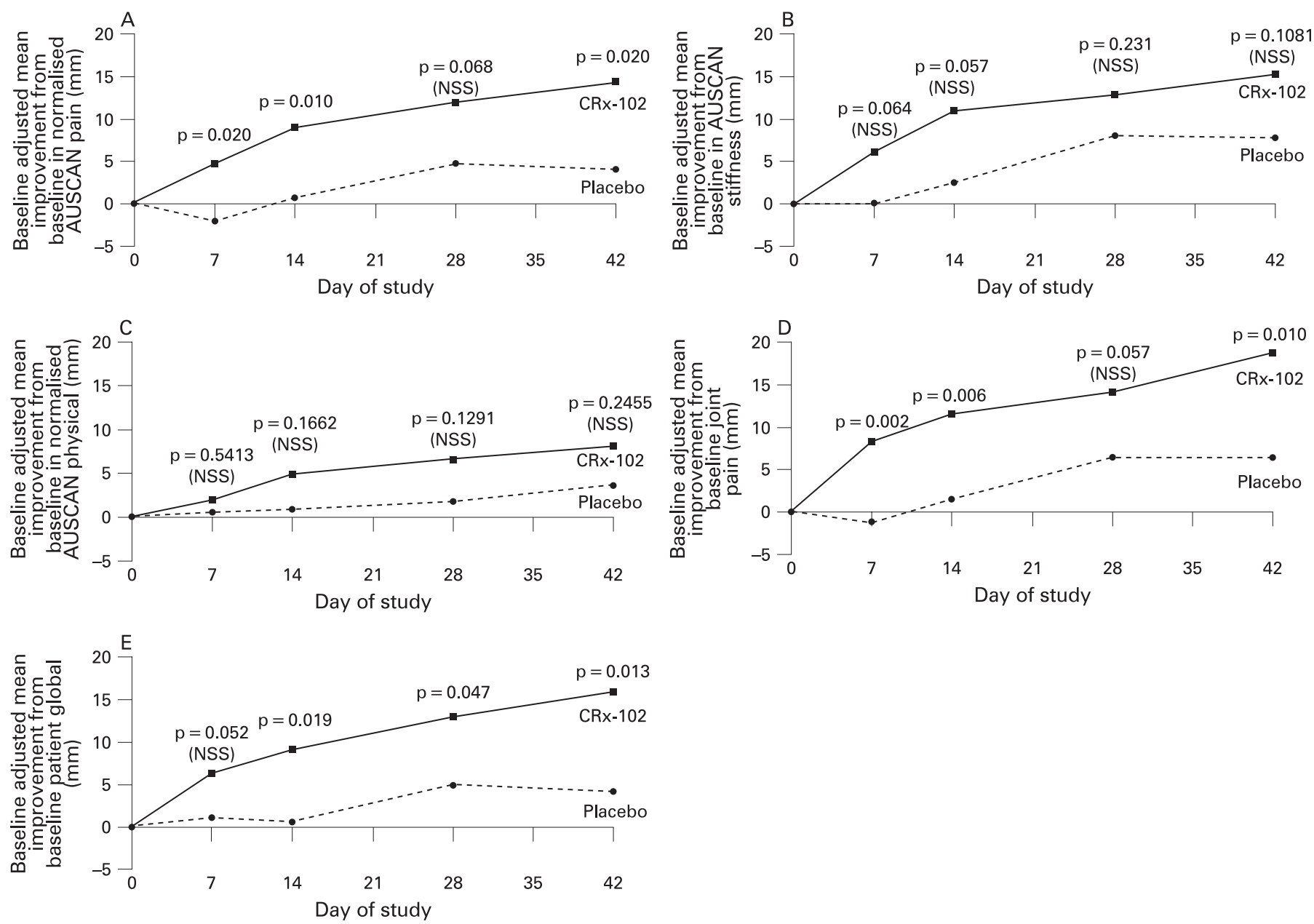

Figure 2 Mean improvements from baseline adjusted for baseline values Australian/Canadian Osteoarthritis Hand Index (AUSCAN) pain (A), AUSCAN stiffness (B), AUSCAN physical (C), pain visual analogue scale (VAS) (D) and global VAS (E) in patients receiving CRx-102 and placebo (intention to treat population) with one-sided $p$ values for the differences of adjusted least square means.

appropriate to use two-sided tests, ie, a more conservative approach, in this work). The statistical analyses were performed by the sponsor in collaboration with the principal investigator (TKK).

\section{RESULTS}

A total of 83 patients (77 (93\%) females) with a mean (standard deviation (SD)) age of 60.4 (5.2) years were enrolled into the study. A Consolidated Standards of Reporting Trials (CONSORT) flow-chart is shown in fig 1 . The study groups were comparable for demographic characteristics (table 1). The patients in the active treatment group had wider OA joint involvement (table 1), but the baseline levels of pain and physical limitations were comparable (table 2).

A significant difference $(p=0.020)$ in the AUSCAN pain score (the primary endpoint) at the end of the study was demonstrated in favour of CRx-102 compared to placebo in the ITT population (table 2). CRx-102 was also statistically superior to placebo at 42 days for joint pain VAS and patient global VAS (table 2). Figure 2 displays how the improvement developed over time and also that the differences between CRx-102 and placebo were discernible after 2 weeks. The comparison between CRx-102 and placebo in the per-protocol population was also consistently in favour of CRx-102 (table 3).

The tender and swollen joint counts of the 18 PIP and DIP joints were numerically improved in the CRx-102 group compared to placebo (table 2) and the group differences approach statistical significance in the per-protocol population (table 3 ). The counts of joints with limited motion and bony swelling did not change during the study (data not shown).

The proportions of patients reporting at least one adverse event in the CRx-102 and placebo groups were 64\% and $32 \%$, respectively. The most common adverse event in both groups was headache, which was more frequently reported in the CRx102 group (52\%) than in the placebo group (15\%). A total of $21 \%$ of the patients in the CRx-102 group also reported nausea, versus none in the placebo group (table 4). No serious adverse events were reported in the CRx-102 group. Discontinuation occurred more often in the CRx-102 ( $\mathrm{n}=16,38 \%)$ than in placebo group ( $n=6,15 \%$ ) (fig 1 ) and was mostly due to headache. Most of the discontinuations occurred early in the study (fig 3).

\section{DISCUSSION}

$\mathrm{HOA}$ is a frequent disease in people more than 60 years of age and imparts a considerable disease burden, on the individual ${ }^{6}$ and in society. ${ }^{2}$ However, few studies have formally addressed the efficacy of symptom-modifying drugs in HOA. ${ }^{10}{ }^{15}$ This phase 2 study demonstrated that CRx-102, a combination of a low dose prednisolone and a titrated dose of dipyridamole, was superior to placebo across a variety of patient-reported measures. 
Table 3 Baseline mean (SD) values of efficacy variables, adjusted mean changes from baseline to day 42 (least squares mean (SEM)) and treatment effect (mean difference (95\% Cl) placebo minus CRx-102) in the per-protocol population

\begin{tabular}{|c|c|c|c|c|c|c|}
\hline & \multicolumn{2}{|l|}{ Baseline } & \multicolumn{2}{|l|}{ Changes } & \multirow[b]{2}{*}{ Treatment effect } & \multirow[b]{2}{*}{ p Value } \\
\hline & CRx-102 (n= 26) & Placebo $(n=33)$ & CRx-102 & Placebo & & \\
\hline \multicolumn{7}{|l|}{ AUSCAN: } \\
\hline Pain & $61.9(16.6)$ & $63.8(17.2)$ & $-20.5(4.1)$ & $-6.2(3.7)$ & 14.3 (3.2 to 25.5$)$ & 0.012 \\
\hline Physical & $64.9(18.9)$ & $70.9(15.5)$ & $-12.9(3.7)$ & $-5.9(3.2)$ & $7.0(-2.9$ to 16.8$)$ & 0.061 \\
\hline Stiffness & $62.9(17.4)$ & $67.8(19.8)$ & $-20.3(4.4)$ & $-8.3(3.9)$ & $12.0(0.2$ to 23.9$)$ & 0.047 \\
\hline \multicolumn{7}{|l|}{ VAS: } \\
\hline Joint pain & $59.8(19.5)$ & $62.9(16.7)$ & $-23.5(4.4)$ & $-6.3(3.9)$ & 17.2 (5.5 to 28.9 ) & 0.005 \\
\hline \multicolumn{7}{|l|}{ Lab tests: } \\
\hline CRP mg/litre & $2.0(1.8)$ & $2.3(2.2)$ & $-0.2(0.5)$ & $0.4(0.4)$ & $0.6(-0.7$ to 1.8$)$ & 0.364 \\
\hline \multicolumn{7}{|l|}{ Joint counts: } \\
\hline Tender joints & $9.6(4.8)$ & $9.8(4.7)$ & $-5.0(1.0)$ & $-2.6(0.9)$ & $2.4(-0.3$ to 5.0$)$ & 0.083 \\
\hline Soft tissue swelling & $5.6(4.6)$ & $4.9(4.3)$ & $-3.1(0.6)$ & $-1.9(0.5)$ & $1.3(-0.3$ to 2.8$)$ & 0.116 \\
\hline
\end{tabular}

AUSCAN, Australian/Canadian Osteoarthritis Hand Index; CRP, C-reactive protein; VAS, visual analogue scale.

Table 4 Most commonly reported adverse events $(\mathrm{AE})(\geqslant 5 \%$ of total subjects) in the intention to treat (ITT) population (no. of patients (\%))

\begin{tabular}{llcc}
\hline & CRx-102 $(\mathbf{n}=\mathbf{4 2})$ & Placebo $(\mathbf{n}=\mathbf{4 1})$ & Total $(\mathbf{n}=\mathbf{8 3})$ \\
\hline Subjects with at least one AE & $27(64)$ & $13(32)$ & $40(48)$ \\
Headache & $22(52)$ & $6(15)$ & $28(34)$ \\
Nausea & $9(21)$ & 0 & $9(11)$ \\
\hline
\end{tabular}

In HOA the DIP and PIP joints as well as the first carpometacarpal (CMC) joint are particularly affected, ${ }^{27}$ but patients with HOA tend to have involvement of multiple joints, a condition that is often referred to as generalised OA. ${ }^{28}$ The joints most frequently involved in this study population were the first metatarsophalangeal (MTP) joint, the knee and additionally the lumbar and cervical spine (table 1). We do not know how CRx-102 influenced these other joint areas, since we were not measuring low back pain, pain in the big toe or were not using the Western Ontario and McMaster Universities (WOMAC) index, ${ }^{29}$ a specific measure of knee and hip OA. However, the question on joint pain did not specifically address pain in the finger joints and the clear differentiation between CRx-102 and placebo for this measure may indirectly support an efficacy that goes beyond the finger joints.

Inflammation has been recognised as a feature of the disease process in $\mathrm{OA} .{ }^{30-33}$ A synergistic drug candidate such as $\mathrm{CRx}-102$ comprises two components that are designed to act synergistically through multiple pathways, providing a novel therapeutic effect that neither component can achieve on their own. CRx-102 has been shown to have strong anti-inflammatory effects in

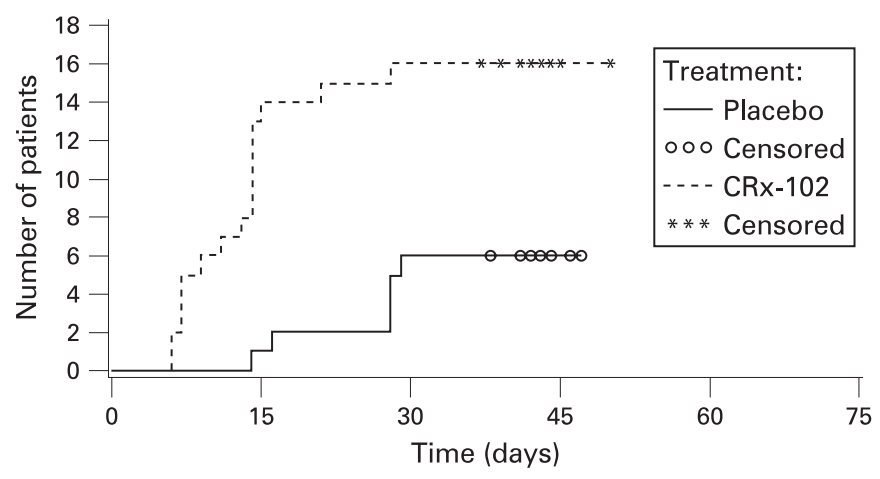

Figure 3 Time from dose 1 to withdrawal from the treatment (intention to treat (ITT) population, Kaplan-Meier plot). preclinical assays with a greater average percentage inhibition of tumour necrosis factor alpha (TNF $\alpha$ ) and interleukin 1 (IL1) release from human blood buffy coat cells than the inhibition seen with each of the single agents alone. ${ }^{20}$ Similarly, in the rat model of lipopolysaccharide (LPS)-induced TNF $\alpha$ release, the inhibition of release was greater with CRx-102 than the individual components. ${ }^{21}$ This finding was replicated in the rat models of adjuvant and collagen-induced arthritis. ${ }^{21}$

The preclinical studies on the mechanism of action of CRx102 indicate that CRx-102 acts through multiple molecular pathways to create a synergistic immunomodulatory effect without amplifying traditional glucocorticoid-associated side effects. Additionally, the components of CRx-102 affect the activity of key transcription factors, but not their nuclear localisation nor does it increase glucocorticoid receptor translocation or transcription from positive glucocorticoid response element promoters relative to low dose prednisolone on its own. ${ }^{22}$ Dipyridamole may contribute to the action of CRx-102 by increasing CAMP in part though inhibition of phosphodiesterases relevant to inflammation, as well as through modulating adenosine transport resulting in increased extra-cellular endogenous adenosine. ${ }^{34}$ Adenosine can suppress the release of TNF $\alpha$ from activated monocytes and macrophages, but a small placebo-controlled study was unable to demonstrate clinical improvement following treatment with dipyridamole. ${ }^{35}$

This study used the pain dimension in AUSCAN as the primary endpoint, but also included an assessment of two other core measures, function and patient global assessment. ${ }^{36}{ }^{37}$ AUSCAN has been developed as a disease-specific patientreported measure in hand OA. Development of the AUSCAN $\mathrm{HOA}$ index was based on the questions and experience from the $\mathrm{WOMAC}^{38}$ as well as information from patient interviews. ${ }^{24}$ AUSCAN was later validated in separate studies ${ }^{25}$ and has also been validated in the Norwegian language. ${ }^{26}$ Consistent with the present findings, Allen et $a^{39}$ showed that the AUSCAN index can reliably measure changes in pain, stiffness and function, thereby providing a meaningful endpoint for clinical trials in $\mathrm{HOA}$. For feasibility reasons we chose not to include 
other hand indexes that are alternatives to AUSCAN in the assessment of HOA. ${ }^{40} 41$

The patients had to stop treatment with NSAIDs and paracetamol at the screening visit, but a disease flare was not required for inclusion in the study. We unfortunately did not capture detailed information about ongoing medication at the screening visit, and we do not have detailed data on the changes in efficacy endpoints from screening to randomisation.

In rheumatoid arthritis, several composite disease measures have been developed over the past years, and these are most often based on joint counts. Similar composite disease specific measures do not exist in HOA. We examined the number of finger joints with tenderness, soft tissue swelling, limited motion and bony swelling at baseline and during follow-up. It is reasonable to assume that tenderness and soft tissue swelling at least in part reflect the inflammatory component of the disease. Joint counts were numerically improved in the CRx-102 treatment group compared to the placebo group (tables 3 and 4). The number of joints with bony swelling did not change during the study; this endpoint was not expected to be influenced by anti-inflammatory therapy. More research is needed to address the development of joint count-indices for $\mathrm{HOA}$ that are valid, reliable and responsive.

Headache was the most commonly reported adverse event in this trial and was most frequently observed during the first days of treatment (table 4 and fig 3). This type of headache has previously been associated with dipyridamole administration ${ }^{42}$ and also with other cardiovascular pharmaceutical products with vasodilatating properties. As in the current study, these headaches typically occur during the first days of treatment. ${ }^{42}$ This early and high withdrawal rate is a potential limitation of CRx-102. Thus, formulation development is necessary to optimise the synergistic benefits of CRx-102 demonstrated in this and other phase 2 studies and to minimise the observed incidence of headache. The objective of the formulation should be to deliver pulsed-doses of prednisolone with concurrently releasing dipyridamole at a rate that maintains the antiinflammatory synergy and minimises the vasodilator effects that are known to cause headaches.

In summary, preclinical studies have supported that the synergistic drug candidate CRx-102, comprised of dipyramidole and low dose prednisolone, has biological effects that exceed the effects of each individual component. This placebo-controlled phase 2 study suggests that the combination is effective in patients with HOA. Follow-up studies should be initiated to compare the clinical effects of CRx-102 versus the individual components in HOA and other rheumatic joint diseases.

Funding: This study was funded by CombinatoRx Inc, the producer of CRx-102.

Competing interests: MN, YZ and JL are employees of CombinatoRx Inc, the producer of CRx-102. The statistical analyses were performed by the sponsor in collaboration with the principal investigator (TKK).

Ethics approval: The regional ethics committee evaluated the study, the storage and analyses of data was licensed from the data inspectorate, and approval for the collection of biologic material was obtained from the Department of Health.

\section{REFERENCES}

1. Ehrlich GE. The rise of osteoarthritis. Bull World Health Organ 2003:81;630.

2. Petersson IF, Jacobsson LT. Osteoarthritis of the peripheral joints. Best Pract Res Clin Rheumatol 2002;16:741-60.

3. Oliveria SA, Felson DT, Reed JI, Cirillo PA, Walker AM. Incidence of symptomatic hand, hip, and knee osteoarthritis among patients in a health maintenance organization. Arthritis Rheum 1995;38:1134-41.

4. Zhang Y, Niu J, Kelly-Hayes M, Chaisson CE, Aliabadi P, Felson DT. Prevalence of symptomatic hand osteoarthritis and its impact on functional status among the elderly: The Framingham Study. Am J Epidemiol 2002;156;1021-7.
5. Dahaghin S, Bierma-Zeinstra SM, Ginai AZ, Pols HA, Hazes JM, Koes BW. Prevalence and pattern of radiographic hand osteoarthritis and association with pain and disability (the Rotterdam study). Ann Rheum Dis 2005;64:682-7.

6. Slatkowsky-Christensen B, Mowinckel P, Loge JH, Kvien TK. Health related quality of life in females with symptomatic hand osteoarthritis - a comparison with female rheumatoid arthritis patients, healthy controls and normative data. Arthritis Rheum 2007; 57:1404-9

7. Zhang W, Doherty M, Arden N, Bannwarth B, Bijlsma J, Gunther KP, et al. EULAR evidence based recommendations for the management of hip osteoarthritis: report of a task force of the EULAR Standing Committee for International Clinical Studies Including Therapeutics (ESCISIT). Ann Rheum Dis 2005;64:669-81.

8. Wegman A, van der WD, van Tulder M, Stalman W, de Vries T. Nonsteroidal antiinflammatory drugs or acetaminophen for osteoarthritis of the hip or knee? A systematic review of evidence and guidelines. J Rheumatol 2004;31:344-54.

9. Jordan KM, Arden NK, Doherty M, Bannwarth B, Bijlsma JW, Dieppe P, et al. EULAR recommendations 2003: an evidence based approach to the management of knee osteoarthritis: report of a task force of the Standing Committee for International Clinical Studies Including Therapeutics (ESCISIT). Ann Rheum Dis 2003; 62:1145-55.

10. Zhang W, Doherty M, Leeb BF, Alekseeva L, Arden NK, Bijlsma JW, et al. EULAR evidence based recommendations for the management of hand osteoarthritis - report of a task force of the Eular Standing Committee for International Clinical Studies Including Therapeutics (ESCISIT). Ann Rheum Dis 2007;66:377-88.

11. Bresalier RS, Sandler RS, Ouan H, Bolognese JA, Oxenius B, Horgan K, et al. Cardiovascular events associated with rofecoxib in a colorectal adenoma chemoprevention trial. N Engl J Med 2005;352:1092-102.

12. Solomon SD, McMurray JJ, Pfeffer MA, Wittes J, Fowler R, Finn P, et al. Cardiovascular risk associated with celecoxib in a clinical trial for colorectal adenoma prevention. N Engl J Med 2005;352:1071-80.

13. McGettigan $\mathbf{P}$, Henry D. Cardiovascular risk and inhibition of cyclooxygenase: a systematic review of the observational studies of selective and nonselective inhibitors of cyclooxygenase 2. JAMA 2006;296:1633-44.

14. Bjordal JM, Ljunggren $A E$, Klovning $A$, et al. Non-steroidal anti-inflammatory drugs, including cyclo-oxygenase-2 inhibitors, in osteoarthritic knee pain: meta-analysis of randomised placebo controlled trials. BMJ 2004;329:1317.

15. Maheu E, Altman RD, Bloch DA, Doherty M, Hochberg M, Mannoni A, et al. Design and conduct of clinical trials in patients with osteoarthritis of the hand: recommendations from a task force of the Osteoarthritis Research Society International. Osteoarthritis Cartilage 2006;14:303-22.

16. Kloppenburg M, Stamm T, Watt I, Kainberger F, Caston TE, Birrell FN, et al. Research in hand osteoarthritis: time for reappraisal and demand for new strategies. Ann Rheum Dis 2007;66: 1157-61.

17. Rhen T, Cidlowski JA. Antiinflammatory action of glucocorticoids - new mechanisms for old drugs. N Engl J Med 2005;353:1711-23.

18. Keith CT, Borisy AA, Stockwell BR. Multicomponent therapeutics for networked systems. Nat Rev Drug Discov 2005;4:71-8.

19. Zimmermann GR, Lehar J, Keith CT. Multi-target therapeutics: when the whole is greater than the sum of the parts. Drug Discov Today 2007;12:34-42.

20. Borisy AA, Elliott PJ, Hurst NW, Lee MS, Lehar J, Price ER, et al. Systematic discovery of multicomponent therapeutics. Proc Natl Acad Sci USA 2003; 100:7977-82.

21. Elliott PJ, Keegan M, Avery W, Borisy AA. Crx-102: A novel, orally available, syncretic agent with significant in vitro and in vivo immuno-modulatory activity and a dissociated steroid profile. Arthritis Rheum 2004;50(Suppl):S370-1.

22. Price ER, Manivasakam P, Robinson E, Slanonic M, Nolan G, Smith B, et al. Molecular insight into steroid dissociation of crx-102, a clinically active immunomodulatory agent. Arthritis Rheum 2006;54(Suppl):S592.

23. Altman R, Alarcon G, Appelrouth D, Bloch D, Borenstein D, Brandt K, et al. The American College of Rheumatology criteria for the classification and reporting of osteoarthritis of the hand. Arthritis Rheum 1990;33:1601-10.

24. Bellamy N, Campbell J, Haraoui B, Buchbinder R, Hobby K, Roth JH, et al. Dimensionality and clinical importance of pain and disability in hand osteoarthritis: Development of the Australian/Canadian (AUSCAN) Osteoarthritis Hand Index. Osteoarthritis Cartilage 2002;10:855-62.

25. Bellamy N, Campbell J, Haraoui B, Gerecz-Simon E, Buchbinder R, Hobby K, et al. Clinimetric properties of the AUSCAN Osteoarthritis Hand Index: an evaluation of reliability, validity and responsiveness. Osteoarthritis Cartilage 2002;10:863-9.

26. Slatkowsky-Christensen B, Kvien TK, Bellamy N. Performance of the Norwegian version of AUSCAN - a disease-specific measure of hand osteoarthritis. Osteoarthritis Cartilage 2005;13:561-7.

27. Niu J, Zhang Y, LaValley M, Chaisson CE, Aliabadi P, Felson DT. Symmetry and clustering of symptomatic hand osteoarthritis in elderly men and women: the Framingham Study. Rheumatology (Oxford) 2003;42:343-8.

28. Dougados M, Nakache JP, Gueguen A. Criteria for generalized and focal osteoarthritis. Rev Rhum Engl Ed 1996;63:569-75.

29. Bellamy N, Buchanan WW, Goldsmith CH, Campbell J, Stitt LW. Validation study of WOMAC: a health status instrument for measuring clinically important patient relevant outcomes to antirheumatic drug therapy in patients with osteoarthritis of the hip or knee. J Rheumatol 1988;15:1833-40.

30. Saxne T, Lindell M, Mansson B, Petersson IF, Heinegard D. Inflammation is a feature of the disease process in early knee joint osteoarthritis. Rheumatology (Oxford) 2003:42:903-4. 
31. Spector TD, Hart DJ, Nandra D, Doyle DV, Mackillop N, Gallimore JR, et al. Low level increases in serum C-reactive protein are present in early osteoarthritis of the knee and predict progressive disease. Arthritis Rheum 1997;40:723-7.

32. Sharif M, Shepstone L, Elson CJ, Dieppe PA, Kirwan JR. Increased serum C reactive protein may reflect events that precede radiographic progression in osteoarthritis of the knee. Ann Rheum Dis 2000;59:71-4.

33. Pelletier JP, Martel-Pelletier J, Abramson SB. Osteoarthritis, an inflammatory disease: potential implication for the selection of new therapeutic targets. Arthritis Rheum 2001;44:1237-47.

34. Riksen NP, Barrera P, van den Broek PH, van Riel PL, Smits P, Rongen GA. Methotrexate modulates the kinetics of adenosine in humans in vivo. Ann Rheum Dis 2006;65:465-70.

35. Forrest CM, Stoy N, Stone TW, Harman G, Mackay GM, Oxford L, et al. Adenosine and cytokine levels following treatment of rheumatoid arthritis with dipyridamole. Rheumatol Int 2006:27:11-7.

36. Altman R, Brandt K, Hochberg M, Moskowitz R, Bellamy N, Bloch DA, et al. Design and conduct of clinical trials in patients with osteoarthritis: recommendations from a task force of the Osteoarthritis Research Society. Results from a workshop. Osteoarthritis Cartilage 1996:4:217-43.
37. Bellamy N, Kirwan J, Boers M, Brooks P, Strand V, Tugwell P, et al. Recommendations for a core set of outcome measures for future phase III clinical trials in knee, hip, and hand osteoarthritis. Consensus development at OMERACT III. J Rheumatol 1997:24:799-802.

38. Bellamy N. The WOMAC Knee and Hip Osteoarthritis Indices: development, validation, globalization and influence on the development of the AUSCAN Hand Osteoarthritis Indices. Clin Exp Rheumatol 2005;23:S148-3.

39. Allen KD, Jordan JM, Renner JB, Kraus VB. Validity, factor structure, and clinical relevance of the AUSCAN Osteoarthritis Hand Index. Arthritis Rheum 2006;54:551-6.

40. Dreiser RL, Maheu E, Guillou GB, Caspard H, Grouin JM. Validation of an algofunctional index for osteoarthritis of the hand. Rev Rhum Engl Ed 1995:62:43S-53S.

41. Leeb BF, Sautner J, Andel I, Rintelen B. SACRAH: a score for assessment and quantification of chronic rheumatic affections of the hands. Rheumatology (Oxford) 2003;42:1173-8.

42. Theis JG, Deichsel G, Marshall S. Rapid development of tolerance to dipyridamoleassociated headaches. Br J Clin Pharmacol 1999:48:750-5.

\section{BMJ Careers online re-launches}

BMJ Careers online has re-launched to give you an even better online experience. You'll still find our online services such as jobs, courses and careers advice, but now they're even easier to navigate and quicker to find.

New features include:

- Job alerts - you tell us how often you want to hear from us with either daily or weekly alerts

- Refined keyword searching making it easier to find exactly what you want

- Contextual display - when you search for articles or courses we'll automatically display job adverts relevant to your search

- Recruiter logos linked directly to their organisation homepage - find out more about the company before you apply

- RSS feeds now even easier to set up

Visit careers.bmj.com to find out more. 